\title{
Existence of nontrivial solutions for fractional Schrödinger equations with electromagnetic fields and critical or supercritical nonlinearity
}

Quanqing Li ${ }^{1}$, Kaimin Teng ${ }^{2}$, Wenbo Wang ${ }^{3}$ and Jian Zhang ${ }^{4,5 *}$

"Correspondence:

zhangjian433130@163.com

${ }^{4}$ School of Mathematics and

Statistics, Hunan University of

Technology and Business,

Changsha, P.R. China

${ }^{5}$ School of Mathematics and

Statistics, Central South University,

Changsha, P.R. China

Full list of author information is

available at the end of the article

\begin{abstract}
In this paper, we study the following fractional Schrödinger equation with electromagnetic fields and critical or supercritical nonlinearity:

$$
(-\Delta)_{A}^{s} u+V(x) u=f\left(x,|u|^{2}\right) u+\lambda|u|^{p-2} u, \quad x \in \mathbb{R}^{N},
$$

where $(-\Delta)_{A}^{s}$ is the fractional magnetic operator with $0<s<1, N>2 s, \lambda>0,2_{s}^{*}=\frac{2 N}{N-2 s}$, $p \geq 2_{s}^{*}, f$ is a subcritical nonlinearity, and $V \in C\left(\mathbb{R}^{N}, \mathbb{R}\right)$ and $A \in C\left(\mathbb{R}^{N}, \mathbb{R}^{N}\right)$ are the electric and magnetic potentials, respectively. Under some suitable conditions, by variational methods we prove that the equation has a nontrivial solution for small $\lambda>0$. Our main contribution is related to the fact that we are able to deal with the case $p>2$.
\end{abstract}

MSC: 35J10; 35J20; 35J50; 35J70; 35P05

Keywords: Fractional Schrödinger equation; Fractional magnetic operator; Critical or supercritical nonlinearity

\section{Introduction and preliminaries}

Consider the following fractional Schrödinger equation with electromagnetic fields and critical or supercritical nonlinearity:

$$
(-\Delta)_{A}^{s} u+V(x) u=f\left(x,|u|^{2}\right) u+\lambda|u|^{p-2} u, \quad x \in \mathbb{R}^{N},
$$

where $(-\Delta)_{A}^{s}$ is the fractional magnetic operator with $0<s<1, N>2 s, \lambda>0,2_{s}^{*}=\frac{2 N}{N-2 s}$, $p \geq 2_{s}^{*}, f$ is a subcritical nonlinearity, and $V \in C\left(\mathbb{R}^{N}, \mathbb{R}\right)$ and $A \in C\left(\mathbb{R}^{N}, \mathbb{R}^{N}\right)$ are the electric and magnetic potentials, respectively.

The fractional magnetic Laplacian is defined by

$$
(-\Delta)_{A}^{s} u(x)=C_{N, s} \lim _{r \rightarrow 0} \int_{B_{r}^{c}(x)} \frac{u(x)-e^{i(x-y) \cdot A\left(\frac{x+y}{2}\right)} u(y)}{|x-y|^{N+2 s}} d y, \quad C_{N, s}=\frac{4^{s} \Gamma\left(\frac{N+2 s}{2}\right)}{\pi^{\frac{N}{2}}|\Gamma(-s)|} .
$$

(c) The Author(s) 2020. This article is licensed under a Creative Commons Attribution 4.0 International License, which permits use sharing, adaptation, distribution and reproduction in any medium or format, as long as you give appropriate credit to the original author(s) and the source, provide a link to the Creative Commons licence, and indicate if changes were made. The images or other third party material in this article are included in the article's Creative Commons licence, unless indicated otherwise in a credit line to the material. If material is not included in the article's Creative Commons licence and your intended use is not permitted by statutory regulation or exceeds the permitted use, you will need to obtain permission directly from the copyright holder. To view a copy of this licence, visit http://creativecommons.org/licenses/by/4.0/. 
This nonlocal operator has been defined in [4] as a fractional extension (for any $s \in(0,1)$ ) of the magnetic pseudorelativistic operator or Weyl pseudodifferential operator defined with midpoint prescription [1]. As stated in [17], up to correcting the operator by the factor $(1-s)$, it follows that $(-\Delta)_{A}^{s} u$ converges to $-(\nabla u-i A)^{2} u$ as $s \rightarrow 1$. Thus, up to normalization, the nonlocal case can be seen as an approximation of the local one. The motivation for its introduction is described in more detail in $[4,17]$ and relies essentially on the Lévy-Khintchine formula for the generator of a general Lévy process.

The main driving force for the study of problem (1.1) arises in the following timedependent Schrödinger equation when $s=1$ :

$$
i \hbar \frac{\partial \psi}{\partial t}=\frac{1}{2 m}(-i \hbar \nabla+A(x))^{2} \psi+P(x) \psi-\rho(x,|\psi|) \psi,
$$

where $\hbar$ is the Planck constant, $m$ is the particle mass, $A: \mathbb{R}^{N} \rightarrow \mathbb{R}^{N}$ is the magnetic potential, $P: \mathbb{R}^{N} \rightarrow \mathbb{R}^{N}$ is the electric potential, $\rho$ is the nonlinear coupling, and $\psi$ is the wave function representing the state of the particle. This equation arises in quantum mechanics and describes the dynamics of the particle in a nonrelativistic setting $[2,15]$. Clearly, the form $\psi(x, t):=e^{-i \varpi t h^{-1}} u(x)$ is a standing wave solution of (1.2) if and only if $u(x)$ satisfies the following stationary equation:

$$
(-i \varepsilon \nabla+A)^{2} u+V(x) u=f(x,|u|) u,
$$

where $\varepsilon=\hbar, V(x)=2 m(P(x)-\varpi)$, and $f=2 m \rho$; see $[3,5,7,8]$. By applying variational methods and Lyusternik-Schnirelmann theory Ambrosio and d'Avenia [1] proved the existence and multiplicity of solutions for the equation

$$
\varepsilon^{2 s}(-\Delta)_{A / \varepsilon}^{s} u+V(x) u=f\left(|u|^{2}\right) u
$$

when $\varepsilon>0$ is small. Recently, Liang et al. [14] obtained the existence and multiplicity of solutions for the fractional Schrödinger-Kirchhoff equation

$$
\varepsilon^{2 s} M\left([u]_{s, A_{\varepsilon}}^{2}\right)(-\Delta)_{A_{\varepsilon}}^{s} u+V(x) u=|u|^{2_{s}^{*}-2} u+h\left(x,|u|^{2}\right) u
$$

with the help of fractional version of the concentration compactness principle and variational methods. If the magnetic field $A \equiv 0$, then the operator $(-\Delta)_{A}^{s}$ can be reduced to the fractional Laplacian operator $(-\Delta)^{s}$, which is defined as

$$
(-\Delta)^{s} u(x)=C_{N, s} \text { P.V. } \int_{\mathbb{R}^{N}} \frac{u(x)-u(y)}{|x-y|^{N+2 s}} d y=C_{N, s} \lim _{\varepsilon \rightarrow 0^{+}} \int_{\mathbb{R}^{N} \backslash B_{\varepsilon}(x)} \frac{u(x)-u(y)}{|x-y|^{N+2 s}} d y .
$$

The symbol P.V. stands for the Cauchy principal value, and $C_{N, s}$ is a dimensional constant that depends on $N, s$, precisely given by

$$
C_{N, s}=\left(\int_{\mathbb{R}^{N}} \frac{1-\cos \zeta_{1}}{|\zeta|^{N+2 s}} d \zeta\right)^{-1}
$$

It is well known that the fractional Laplacian $(-\Delta)^{s}$ can be viewed as a pseudodifferential operator of symbol $|\xi|^{2 s}$, as stated in Lemma 1.1 in [6]. Simultaneously, problem (1.1) be- 
comes the classical Schrödinger equation

$$
(-\Delta)^{s} u+V(x) u=f\left(x,|u|^{2}\right) u+\lambda|u|^{p-2} u, \quad x \in \mathbb{R}^{N} .
$$

Recently, there has been a lot of interest in the study of equation (1.3) and other related nonlocal problems. See, for instance, $[6,10-13,16,21-23]$ and the references therein. For more results about dealing with magnetic operators, see $[9,20]$. Nonlocal problems also appear in other mathematical research fields. We refer the interested readers to [18, 19] for mathematical researches on Kirchhoff-type nonlocal equations, where Tang and Cheng [19] proposed a new approach to recover compactness for the (PS)-sequence, and Tang and Chen [18] proposed a new approach to recover compactness for the minimizing sequence.

Most of the works mentioned are set in $\mathbb{R}^{N}, N>2 s$, with subcritical or critical growth, and to the best of our knowledge, no results are available on the existence for problem (1.1) with supercritical exponent. In this paper, we aim at studying the existence of nontrivial solutions for critical or supercritical problem (1.1).

To reduce the statements of the main result, we introduce the following assumptions:

(V) $V \in C\left(\mathbb{R}^{N}, \mathbb{R}\right), 0<V_{0}:=\inf _{x \in \mathbb{R}^{N}} V(x)$, and $\lim _{|x| \rightarrow+\infty} V(x)=+\infty$.

$\left(f_{1}\right) f \in C\left(\mathbb{R}^{N} \times \mathbb{R}, \mathbb{R}\right)$, and there exists $2<q<2_{s}^{*}$ such that

$$
|f(x, t)| \leq C\left(1+|t|^{\frac{q-2}{2}}\right)
$$

for all $(x, t) \in \mathbb{R}^{N} \times \mathbb{R}$, where $C$ is a positive constant.

$\left(f_{2}\right) f(x, t)=o(1)$ as $|t| \rightarrow 0$ uniformly in $x \in \mathbb{R}^{N}$;

$\left(f_{3}\right) f(x, t) t \geq \frac{q}{2} F(x, t):=\frac{q}{2} \int_{0}^{t} f(x, \tau) d \tau$ for all $(x, t) \in \mathbb{R}^{N} \times \mathbb{R}$;

$\left(f_{4}\right) c_{0}:=\inf _{x \in \mathbb{R}^{N},|t|=1} F(x, t)>0$.

For a function $u: \mathbb{R}^{N} \rightarrow \mathbb{C}$, we set

$$
[u]_{A}^{2}=\int_{\mathbb{R}^{2 N}} \frac{\left|u(x)-e^{i(x-y) \cdot A\left(\frac{x+y}{2}\right)} u(y)\right|^{2}}{|x-y|^{N+2 s}} d x d y
$$

and

$$
D_{A}^{s}\left(\mathbb{R}^{N}, \mathbb{C}\right)=\left\{u \in L^{2_{s}^{*}}\left(\mathbb{R}^{N}, \mathbb{C}\right):[u]_{A}^{2}<+\infty\right\} .
$$

Then we may introduce the Hilbert space

$$
H_{A}^{s}\left(\mathbb{R}^{N}, \mathbb{C}\right)=\left\{u \in D_{A}^{s}\left(\mathbb{R}^{N}, \mathbb{C}\right): \int_{\mathbb{R}^{N}}|u|^{2} d x<+\infty\right\}
$$

endowed with the scalar product

$$
\begin{aligned}
\langle u, v\rangle_{A}:= & \frac{C_{N, s}}{2} \mathcal{R} \int_{\mathbb{R}^{2 N}} \frac{\left[u(x)-e^{i(x-y) \cdot A\left(\frac{x+y}{2}\right)} u(y)\right] \cdot \overline{\left[v(x)-e^{i(x-y) \cdot A\left(\frac{x+y}{2}\right)} v(y)\right]}}{|x-y|^{N+2 s}} d x d y \\
& +\mathcal{R} \int_{\mathbb{R}^{N}} u \bar{\nu} d x
\end{aligned}
$$


and norm

$$
\|u\|_{A}^{2}=\langle u, u\rangle_{A}
$$

where $\mathcal{R}(z)$ is the real part of a complex number $z$. By Lemma 3.5 in [4] the embedding $H_{A}^{s}\left(\mathbb{R}^{N}, \mathbb{C}\right) \hookrightarrow L^{t}\left(\mathbb{R}^{N}, \mathbb{C}\right)$ is continuous for any $t \in\left[2,2_{s}^{*}\right]$, and the embedding $H_{A}^{s}\left(\mathbb{R}^{N}, \mathbb{C}\right) \hookrightarrow L_{\text {loc }}^{t}\left(\mathbb{R}^{N}, \mathbb{C}\right)$ is compact for any $t \in\left[1,2_{s}^{*}\right)$. Moreover, set

$$
E=\left\{u \in H_{A}^{s}\left(\mathbb{R}^{N}, \mathbb{C}\right): \int_{\mathbb{R}^{N}} V(x)|u|^{2} d x<+\infty\right\}
$$

with the norm

$$
\|u\|^{2}=\frac{C_{N, s}}{2}[u]_{A}^{2}+\int_{\mathbb{R}^{N}} V(x)|u|^{2} d x
$$

By assumption $(V)$ the embedding $E \hookrightarrow H_{A}^{s}\left(\mathbb{R}^{N}, \mathbb{C}\right)$ is continuous.

For convenience, we define the homogeneous fractional Sobolev space

$$
\mathcal{D}^{s, 2}\left(\mathbb{R}^{N}\right):=\left\{u \in L^{2_{s}^{*}}\left(\mathbb{R}^{N}\right):|\xi|^{s} \hat{u}(\xi) \in L^{2}\left(\mathbb{R}^{N}\right)\right\}
$$

which is the completion of $C_{0}^{\infty}\left(\mathbb{R}^{N}\right)$ under the norm

$$
\|u\|_{\mathcal{D}^{s, 2}\left(\mathbb{R}^{N)}\right.}^{2}:=\int_{\mathbb{R}^{N}}\left|(-\Delta)^{\frac{s}{2}} u\right|^{2} d x=\int_{\mathbb{R}^{N}}|\xi|^{2 s}|\hat{u}(\xi)|^{2} d \xi
$$

Define the norm on $H^{s}\left(\mathbb{R}^{N}\right)$ as follows:

$$
\|u\|_{H^{s}\left(\mathbb{R}^{N}\right)}:=\left[\int_{\mathbb{R}^{N}}|\xi|^{2 s}|\hat{u}(\xi)|^{2} d \xi+\int_{\mathbb{R}^{N}} u^{2} d x\right]^{\frac{1}{2}}=\left[\|u\|_{\mathcal{D}^{s, 2}\left(\mathbb{R}^{N}\right)}^{2}+\|u\|_{L^{2}\left(\mathbb{R}^{N}\right)}^{2}\right]^{\frac{1}{2}}
$$

Moreover, the best fractional critical Sobolev constant is given by

$$
S:=\inf _{u \in \mathcal{D}^{s, 2}\left(\mathbb{R}^{N}\right) \backslash\{0\}} \frac{\|u\|_{\mathcal{D}^{s, 2}\left(\mathbb{R}^{N}\right)}^{2}}{\|u\|_{2_{s}^{*}}^{2}} .
$$

Our main result is the following:

Theorem 1.1 Suppose that $(V)$ and $\left(f_{1}\right)-\left(f_{4}\right)$ are satisfied. Then there exists $\lambda_{0}>0$ such that for each $\lambda \in\left(0, \lambda_{0}\right]$, problem (1.1) has a nontrivial solution $u_{\lambda}$.

As a complement of Theorem 1.1, by the Pohozaev identity we can deduce that the equation

$$
(-\Delta)_{A}^{s} u+\mu u=\lambda|u|^{p-2} u, \quad x \in \mathbb{R}^{N}
$$

with $p \geq 2 *$ and $\mu>0$ has no nontrivial solution for all $\lambda>0$. Indeed, let $u \in E$ be a weak solution of the problem. Then we have the following Pohozaev identity:

$$
\frac{1}{2_{s}^{*}} \cdot \frac{C_{N, s}}{2} \int_{\mathbb{R}^{2 N}} \frac{\left|u(x)-e^{i(x-y) \cdot A\left(\frac{x+y}{2}\right)} u(y)\right|^{2}}{|x-y|^{N+2 s}} d x d y+\frac{1}{2} \mu \int_{\mathbb{R}^{N}}|u|^{2} d x=\frac{\lambda}{p} \int_{\mathbb{R}^{N}}|u|^{p} d x .
$$


Moreover, taking $u$ as the test function, we have

$$
\frac{C_{N, s}}{2} \int_{\mathbb{R}^{2 N}} \frac{\left|u(x)-e^{i(x-y) \cdot A\left(\frac{x+y}{2}\right)} u(y)\right|^{2}}{|x-y|^{N+2 s}} d x d y+\mu \int_{\mathbb{R}^{N}} u^{2} d x=\lambda \int_{\mathbb{R}^{N}}|u|^{p} d x .
$$

Taking into account (1.4) and (1.5), we can derive that

$$
\frac{p-2_{s}^{*}}{2_{s}^{*}} \cdot \frac{C_{N, s}}{2} \int_{\mathbb{R}^{2 N}} \frac{\left|u(x)-e^{i(x-y) \cdot A\left(\frac{x+y}{2}\right)} u(y)\right|^{2}}{|x-y|^{N+2 s}} d x d y+\frac{p-2}{2} \mu \int_{\mathbb{R}^{N}} u^{2} d x=0,
$$

which implies the conclusion.

\section{Proof of Theorem 1.1}

It is well known that a weak solution of problem (1.1) is a critical point of the following functional:

$$
\begin{aligned}
I_{\lambda}(u)= & \frac{1}{2}\|u\|^{2}-\frac{1}{2} \int_{\mathbb{R}^{N}} F\left(x,|u|^{2}\right) d x-\frac{\lambda}{p} \int_{\mathbb{R}^{N}}|u|^{p} d x \\
= & \frac{1}{2} \cdot \frac{C_{N, s}}{2}[u]_{A}^{2}+\frac{1}{2} \int_{\mathbb{R}^{N}} V(x)|u|^{2} d x-\frac{1}{2} \int_{\mathbb{R}^{N}} F\left(x,|u|^{2}\right) d x-\frac{\lambda}{p} \int_{\mathbb{R}^{N}}|u|^{p} d x \\
= & \frac{1}{2} \cdot \frac{C_{N, s}}{2} \int_{\mathbb{R}^{2 N}} \frac{\left|u(x)-e^{i(x-y) \cdot A\left(\frac{x+y}{2}\right)} u(y)\right|^{2}}{|x-y|^{N+2 s}} d x d y+\frac{1}{2} \int_{\mathbb{R}^{N}} V(x)|u|^{2} d x \\
& -\frac{1}{2} \int_{\mathbb{R}^{N}} F\left(x,|u|^{2}\right) d x-\frac{\lambda}{p} \int_{\mathbb{R}^{N}}|u|^{p} d x .
\end{aligned}
$$

Clearly, we cannot apply variational methods directly because the functional $I_{\lambda}$ is not well defined on $E$ unless $p=2_{s}^{*}$. To overcome this difficulty, we define the function

$$
\phi(t)= \begin{cases}|t|^{p-2} t & \text { if }|t| \leq M \\ M^{p-q}|t|^{q-2} t & \text { if }|t|>M\end{cases}
$$

where $M>0$. Then $\phi \in C(\mathbb{R}, \mathbb{R}), \phi(t) t \geq q \Phi(t):=q \int_{0}^{t} \phi(\tau) d \tau \geq 0$, and $|\phi(t)| \leq M^{p-q}|t|^{q-1}$ for all $t \in \mathbb{R}$. Set $h_{\lambda}(x, t)=\lambda \phi(t)+f\left(x,|t|^{2}\right) t$ for $(x, t) \in \mathbb{R}^{N} \times \mathbb{R}$. Then $h_{\lambda}(x, t)$ admits the following properties:

$\left(h_{1}\right) \quad h_{\lambda} \in C\left(\mathbb{R}^{N} \times \mathbb{R}, \mathbb{R}\right)$, and $\left|h_{\lambda}(x, t)\right| \leq \lambda M^{p-q}|t|^{q-1}+C\left(|t|+|t|^{q-1}\right)$ for all $(x, t) \in \mathbb{R}^{N} \times \mathbb{R}$.

$\left(h_{2}\right) h_{\lambda}(x, t) t \geq q H_{\lambda}(x, t):=q \int_{0}^{t} h_{\lambda}(x, \tau) d \tau \geq 0$ for all $(x, t) \in \mathbb{R}^{N} \times \mathbb{R}$.

$\left(h_{3}\right) \inf _{x \in \mathbb{R}^{N},|t|=1} H_{\lambda}(x, t) \geq \frac{c_{0}}{2}>0$.

Let

$$
\begin{aligned}
J_{\lambda}(u) & =\frac{1}{2}\|u\|^{2}-\int_{\mathbb{R}^{N}} H_{\lambda}(x, u) d x \\
& =\frac{1}{2} \cdot \frac{C_{N, s}}{2}[u]_{A}^{2}+\frac{1}{2} \int_{\mathbb{R}^{N}} V(x)|u|^{2} d x-\lambda \int_{\mathbb{R}^{N}} \Phi(u) d x-\frac{1}{2} \int_{\mathbb{R}^{N}} F\left(x,|u|^{2}\right) d x .
\end{aligned}
$$

By $\left(h_{1}\right)-\left(h_{3}\right),(V)$, and the mountain pass theorem, using a standard argument, we easily see that the equation

$$
(-\Delta)_{A}^{s} u+V(x) u=h_{\lambda}(x, u)
$$


has a nontrivial solution $u_{\lambda} \in E$ with $J_{\lambda}^{\prime}\left(u_{\lambda}\right)=0$ and $J_{\lambda}\left(u_{\lambda}\right)=c_{\lambda}:=\inf _{\gamma \in \Gamma_{\lambda}} \sup _{t \in[0,1]} J_{\lambda}(\gamma(t))$, where

$$
\Gamma_{\lambda}:=\left\{\gamma \in C([0,1], E): \gamma(0)=0, J_{\lambda}(\gamma(1))<0\right\} .
$$

We further set

$$
\begin{aligned}
J(u) & =\frac{1}{2}\|u\|^{2}-\frac{1}{2} \int_{\mathbb{R}^{N}} F\left(x,|u|^{2}\right) d x \\
& =\frac{1}{2} \cdot \frac{C_{N, s}}{2}[u]_{A}^{2}+\frac{1}{2} \int_{\mathbb{R}^{N}} V(x)|u|^{2} d x-\frac{1}{2} \int_{\mathbb{R}^{N}} F\left(x,|u|^{2}\right) d x, \\
\Gamma & :=\{\gamma \in C([0,1], E): \gamma(0)=0, J(\gamma(1))<0\}
\end{aligned}
$$

and

$$
c:=\inf _{\gamma \in \Gamma} \sup _{t \in[0,1]} J(\gamma(t))
$$

Then $\Gamma \subset \Gamma_{\lambda}$ and $c_{\lambda} \leq c$.

Lemma 2.1 The solution $u_{\lambda}$ satisfies $\left\|u_{\lambda}\right\|^{2} \leq \frac{2 q}{q-2} c_{\lambda}$, and there exists a constant $A>0$ independent on $\lambda$ such that $\left\|u_{\lambda}\right\|^{2} \leq A$.

Proof By $\left(h_{2}\right)$ we know that

$$
\begin{aligned}
q c_{\lambda}= & q J_{\lambda}\left(u_{\lambda}\right)=q J_{\lambda}\left(u_{\lambda}\right)-\left\langle J_{\lambda}^{\prime}\left(u_{\lambda}\right), u_{\lambda}\right\rangle \\
= & \left(\frac{q}{2}-1\right) \cdot \frac{C_{N, s}}{2}\left[u_{\lambda}\right]_{A}^{2}+\left(\frac{q}{2}-1\right) \int_{\mathbb{R}^{N}} V(x)\left|u_{\lambda}\right|^{2} d x \\
& +\int_{\mathbb{R}^{N}}\left[h_{\lambda}\left(x, u_{\lambda}\right) u_{\lambda}-q H_{\lambda}\left(x, u_{\lambda}\right)\right] d x \\
\geq & \left(\frac{q}{2}-1\right)\left\|u_{\lambda}\right\|^{2},
\end{aligned}
$$

which means that $\left\|u_{\lambda}\right\|^{2} \leq \frac{2 q}{q-2} c_{\lambda} \leq \frac{2 q}{q-2} c:=A>0$. This completes the proof.

Lemma 2.2 There exist two constants $B, D>0$ independent on $\lambda$ such that $\left\|\left|u_{\lambda}\right|\right\|_{\infty} \leq B(1+$ $\lambda)^{D}$.

Proof For any $L>0$ and $\beta>1$, set $\gamma(a)=a a_{L}^{2(\beta-1)}, a \in \mathbb{R}$, where $a_{L}:=\min \{|a|, L\}$. Since $\gamma$ is an increasing function, we have

$$
(a-b)[\gamma(a)-\gamma(b)] \geq 0, \quad \forall a, b \in \mathbb{R} .
$$

Let $\Phi(t)=\frac{|t|^{2}}{2}$ and $\Gamma(t)=\int_{0}^{t}\left(\gamma^{\prime}(\tau)\right)^{\frac{1}{2}} d \tau$ for $t \geq 0$. Then if $a>b$, then we have

$$
\Phi^{\prime}(a-b)[\gamma(a)-\gamma(b)]=(a-b)[\gamma(a)-\gamma(b)]=(a-b) \int_{b}^{a} \gamma^{\prime}(t) d t
$$




$$
\begin{aligned}
& =(a-b) \int_{b}^{a}\left(\Gamma^{\prime}(t)\right)^{2} d t \geq\left(\int_{b}^{a} \Gamma^{\prime}(t) d t\right)^{2} \\
& =|\Gamma(a)-\Gamma(b)|^{2} .
\end{aligned}
$$

If $a \leq b$, then we can use a similar argument to obtain the conclusion. It follows that

$$
(a-b)[\gamma(a)-\gamma(b)] \geq|\Gamma(a)-\Gamma(b)|^{2}
$$

for all $a, b \in \mathbb{R}$, which implies that

$$
\begin{aligned}
& \left|\Gamma\left(\left|u_{\lambda}(x)\right|\right)-\Gamma\left(\left|u_{\lambda}(y)\right|\right)\right|^{2} \\
& \quad \leq\left[\left|u_{\lambda}(x)\right|-\left|u_{\lambda}(y)\right|\right] \cdot\left[\left(\left|u_{\lambda}\right| u_{\lambda, L}^{2(\beta-1)}\right)(x)-\left(\left|u_{\lambda}\right| u_{\lambda, L}^{2(\beta-1)}\right)(y)\right] .
\end{aligned}
$$

Choosing $u_{\lambda} u_{\lambda, L}^{2(\beta-1)}$ as a test function, where $u_{\lambda, L}:=\min \left\{\left|u_{\lambda}\right|, L\right\}$, we obtain

$$
\begin{aligned}
\mathcal{R} & {\left[\int_{\mathbb{R}^{N}}\left[f\left(x,\left|u_{\lambda}\right|^{2}\right) u_{\lambda}+\lambda \phi\left(u_{\lambda}\right)\right] \overline{u_{\lambda} u_{\lambda, L}^{2(\beta-1)}} d x\right] } \\
= & \frac{C_{N, s}}{2} \mathcal{R} \\
& \quad \times \int_{\mathbb{R}^{2 N}} \frac{\left[u_{\lambda}(x)-e^{i(x-y) \cdot A\left(\frac{x+y}{2}\right)} u_{\lambda}(y)\right] \cdot \overline{\left[\left(u_{\lambda} u_{\lambda, L}^{2(\beta-1)}\right)(x)-e^{i(x-y) \cdot A\left(\frac{x+y}{2}\right)}\left(u_{\lambda} u_{\lambda, L}^{2(\beta-1)}\right)(y)\right]}}{|x-y|^{N+2 s}} d x d y \\
& +\int_{\mathbb{R}^{N}} V(x)\left|u_{\lambda}\right|^{2} u_{\lambda, L}^{2(\beta-1)} d x .
\end{aligned}
$$

Note that

$$
\begin{aligned}
{\left[u_{\lambda}(x)-e^{i(x-y) \cdot A\left(\frac{x+y}{2}\right)} u_{\lambda}(y)\right] \cdot \overline{\left[\left(u_{\lambda} u_{\lambda, L}^{2(\beta-1)}\right)(x)-e^{i(x-y) \cdot A\left(\frac{x+y}{2}\right)}\left(u_{\lambda} u_{\lambda, L}^{2(\beta-1)}\right)(y)\right]} } \\
=\left[u_{\lambda}(x)-e^{i(x-y) \cdot A\left(\frac{x+y}{2}\right)} u_{\lambda}(y)\right] \cdot\left[\overline{u_{\lambda}(x)} u_{\lambda, L}^{2(\beta-1)}(x)-e^{-i(x-y) \cdot A\left(\frac{x+y}{2}\right)} \overline{u_{\lambda}(y)} u_{\lambda, L}^{2(\beta-1)}(y)\right] \\
=\left|u_{\lambda}(x)\right|^{2} u_{\lambda, L}^{2(\beta-1)}(x)-u_{\lambda}(x) \overline{u_{\lambda}(y)} e^{-i(x-y) \cdot A\left(\frac{x+y}{2}\right)} u_{\lambda, L}^{2(\beta-1)}(y) \\
\quad-u_{\lambda}(y) \overline{u_{\lambda}(x)} e^{i(x-y) \cdot A\left(\frac{x+y}{2}\right)} u_{\lambda, L}^{2(\beta-1)}(x)+\left|u_{\lambda}(y)\right|^{2} u_{\lambda, L}^{2(\beta-1)}(y) \\
\geq\left|u_{\lambda}(x)\right|^{2} u_{\lambda, L}^{2(\beta-1)}(x)-\left|u_{\lambda}(x)\right|\left|u_{\lambda}(y)\right| u_{\lambda, L}^{2(\beta-1)}(y)-\left|u_{\lambda}(y)\right|\left|u_{\lambda}(x)\right| u_{\lambda, L}^{2(\beta-1)}(x) \\
\quad+\left|u_{\lambda}(y)\right|^{2} u_{\lambda, L}^{2(\beta-1)}(y) \\
=\left[\left|u_{\lambda}(x)\right|-\left|u_{\lambda}(y)\right|\right] \cdot\left[\left|u_{\lambda}(x)\right| u_{\lambda, L}^{2(\beta-1)}(x)-\left|u_{\lambda}(y)\right| u_{\lambda, L}^{2(\beta-1)}(y)\right] .
\end{aligned}
$$

Consequently, by (2.1) we have

$$
\begin{aligned}
\mathcal{R} & {\left[\int_{\mathbb{R}^{N}}\left[f\left(x,\left|u_{\lambda}\right|^{2}\right) u_{\lambda}+\lambda \phi\left(u_{\lambda}\right)\right] \overline{u_{\lambda} u_{\lambda, L}^{2(\beta-1)}} d x\right] } \\
\geq & \frac{C_{N, s}}{2} \int_{\mathbb{R}^{2 N}} \frac{\left[\left|u_{\lambda}(x)\right|-\left|u_{\lambda}(y)\right|\right] \cdot\left[\left|u_{\lambda}(x)\right| u_{\lambda, L}^{2(\beta-1)}(x)-\left|u_{\lambda}(y)\right| u_{\lambda, L}^{2(\beta-1)}(y)\right]}{|x-y|^{N+2 s}} d x d y \\
& +\int_{\mathbb{R}^{N}} V(x)\left|u_{\lambda}\right|^{2} u_{\lambda, L}^{2(\beta-1)} d x
\end{aligned}
$$




$$
\geq \frac{C_{N, s}}{2} \int_{\mathbb{R}^{2 N}} \frac{\left|\Gamma\left(\left|u_{\lambda}(x)\right|\right)-\Gamma\left(\left|u_{\lambda}(y)\right|\right)\right|^{2}}{|x-y|^{N+2 s}} d x d y+\int_{\mathbb{R}^{N}} V(x)\left|u_{\lambda}\right|^{2} u_{\lambda, L}^{2(\beta-1)} d x .
$$

For any $\varepsilon>0$, by $\left(f_{1}\right)-\left(f_{2}\right)$ and properties of $\phi$, there exists $C_{\varepsilon}>0$ such that

$$
\left|f\left(x,|t|^{2}\right)\right| \leq \varepsilon+C_{\varepsilon}|t|^{q-2}
$$

and

$$
|\phi(t)| \leq \varepsilon|t|+C_{\varepsilon}|t|^{q-1}
$$

for all $(x, t) \in \mathbb{R}^{N} \times \mathbb{R}$. Thereby, for fixed $\lambda>0$ and small $\varepsilon>0$, we have

$$
\left|f\left(x,|t|^{2}\right) t+\lambda \phi(t)\right| \leq V_{0}|t|+(1+\lambda) C|t|^{q-1}
$$

for all $(x, t) \in \mathbb{R}^{N} \times \mathbb{R}$. Simultaneously, $\Gamma\left(\left|u_{\lambda}\right|\right) \geq \frac{1}{\beta}\left|u_{\lambda}\right| u_{\lambda, L}^{\beta-1}$, and

$$
\frac{C_{N, s}}{2}\left[\Gamma\left(\left|u_{\lambda}\right|\right)\right]_{H^{s}\left(\mathbb{R}^{N}\right)}^{2}=\left\|\Gamma\left(\left|u_{\lambda}\right|\right)\right\|_{\mathcal{D}^{s, 2}\left(\mathbb{R}^{N}\right)}^{2} \geq S\left\|\Gamma\left(\left|u_{\lambda}\right|\right)\right\|_{2_{s}^{*}}^{2} \geq \frac{1}{\beta^{2}} S\left\|\left|u_{\lambda}\right| u_{\lambda, L}^{\beta-1}\right\|_{2_{s}^{*}}^{2} .
$$

Therefore, taking into account (2.2)-(2.4) and condition $(V)$, we can see that

$$
\begin{aligned}
& \frac{1}{\beta^{2}} S\left\|\left|u_{\lambda}\right| u_{\lambda, L}^{\beta-1}\right\|_{2_{s}^{*}}^{2} \\
& \quad \leq \frac{C_{N, s}}{2}\left[\Gamma\left(\left|u_{\lambda}\right|\right)\right]_{H^{s}\left(\mathbb{R}^{N}\right)}^{2} \\
& \quad \leq \mathcal{R} \int_{\mathbb{R}^{N}}\left[f\left(x,\left|u_{\lambda}\right|^{2}\right) u_{\lambda}+\lambda \phi\left(u_{\lambda}\right)\right] \overline{u_{\lambda} u_{\lambda, L}^{2(\beta-1)}} d x-\int_{\mathbb{R}^{N}} V(x)\left|u_{\lambda}\right|^{2} u_{\lambda, L}^{2(\beta-1)} d x \\
& \quad \leq \int_{\mathbb{R}^{N}} V_{0}\left|u_{\lambda}\right|^{2} u_{\lambda, L}^{2(\beta-1)} d x+(1+\lambda) C \int_{\mathbb{R}^{N}}\left|u_{\lambda}\right|^{q} u_{\lambda, L}^{2(\beta-1)} d x-\int_{\mathbb{R}^{N}} V(x)\left|u_{\lambda}\right|^{2} u_{\lambda, L}^{2(\beta-1)} d x \\
& \quad \leq C(1+\lambda) \int_{\mathbb{R}^{N}}\left|u_{\lambda}\right|^{q} u_{\lambda, L}^{2(\beta-1)} d x,
\end{aligned}
$$

which implies that

$$
\left\|\left|u_{\lambda}\right| u_{\lambda, L}^{\beta-1}\right\|_{2_{s}^{*}}^{2} \leq C(1+\lambda) \beta^{2} \int_{\mathbb{R}^{N}}\left|u_{\lambda}\right|^{q} u_{\lambda, L}^{2(\beta-1)} d x
$$

Setting $w_{\lambda, L}=\left|u_{\lambda}\right| u_{\lambda, L}^{\beta-1}$, by the Hölder inequality we can derive that

$$
\begin{aligned}
\left\|w_{\lambda, L}\right\|_{2_{s}^{*}}^{2} & \leq C(1+\lambda) \beta^{2} \int_{\mathbb{R}^{N}}\left|u_{\lambda}\right|^{q-2}\left|u_{\lambda}\right|^{2} u_{\lambda, L}^{2(\beta-1)} d x \\
& \leq C(1+\lambda) \beta^{2}\left(\int_{\mathbb{R}^{N}}\left|u_{\lambda}\right|^{2_{s}^{*}} d x\right)^{\frac{q-2}{2_{s}^{*}}} \cdot\left(\int_{\mathbb{R}^{N}}\left|w_{\lambda, L}\right|^{\alpha_{s}^{*}} d x\right)^{\frac{2}{\alpha_{s}^{*}}},
\end{aligned}
$$

where $\alpha_{s}^{*}=\frac{22_{s}^{*}}{2_{s}^{*}-(q-2)} \in\left(2,2_{s}^{*}\right)$.

By Lemma 2.1 we have

$$
\left\|w_{\lambda, L}\right\|_{2_{s}^{*}}^{2} \leq C(1+\lambda) \beta^{2}\left\|w_{\lambda, L}\right\|_{\alpha_{s}^{*}}^{2}
$$


Now we observe that if $\left|u_{\lambda}\right|^{\beta} \in L^{\alpha_{s}^{*}}\left(\mathbb{R}^{N}\right)$, then from the definition of $\left\{u_{\lambda, L}\right\}$, the inequality $u_{\lambda, L} \leq\left|u_{\lambda}\right|$, and (2.5) we obtain

$$
\left\|w_{\lambda, L}\right\|_{2_{s}^{*}}^{2} \leq C(1+\lambda) \beta^{2}\left(\int_{\mathbb{R}^{N}}\left|u_{\lambda}\right|^{\beta \alpha_{s}^{*}} d x\right)^{\frac{2}{\alpha_{s}^{*}}}<+\infty
$$

Passing to the limit in (2.6) as $L \rightarrow+\infty$, by the Fatou lemma we deduce that

$$
\left\|\left|u_{\lambda}\right|\right\|_{\beta 2_{s}^{*}} \leq C^{\frac{1}{\beta}}(\sqrt{1+\lambda})^{\frac{1}{\beta}} \beta^{\frac{1}{\beta}}\left\|\left|u_{\lambda}\right|\right\|_{\beta \alpha_{s}^{*}}
$$

whenever $\left|u_{\lambda}\right|^{\beta \alpha_{s}^{*}} \in L^{1}\left(\mathbb{R}^{N}\right)$.

Now set $\beta:=\frac{2_{s}^{*}}{\alpha_{s}^{*}}>1$. Since $\left|u_{\lambda}\right| \in L^{2_{s}^{*}}\left(\mathbb{R}^{N}\right)$, the inequality holds for this choice of $\beta$. Then, since $\beta^{2} \alpha_{s}^{*}=\beta 2_{s}^{*}$, it follows that (2.7) holds with $\beta$ replaced by $\beta^{2}$. Consequently,

$$
\begin{aligned}
\left\|\left|u_{\lambda}\right|\right\|_{\beta^{2} 2_{s}^{*}} & \leq C^{\frac{1}{\beta^{2}}}(\sqrt{1+\lambda})^{\frac{1}{\beta^{2}}} \beta^{\frac{2}{\beta^{2}}}\left\|\left|u_{\lambda}\right|\right\|_{\beta^{2} \alpha_{s}^{*}} \\
& =C^{\frac{1}{\beta^{2}}}(\sqrt{1+\lambda})^{\frac{1}{\beta^{2}}} \beta^{\frac{2}{\beta^{2}}}\left\|\left|u_{\lambda}\right|\right\|_{\beta 2_{s}^{*}} \\
& \leq C^{\frac{1}{\beta^{2}}}(\sqrt{1+\lambda})^{\frac{1}{\beta^{2}}} \beta^{\frac{2}{\beta^{2}}} C^{\frac{1}{\beta}}(\sqrt{1+\lambda})^{\frac{1}{\beta}} \beta^{\frac{1}{\beta}}\left\|\left|u_{\lambda}\right|\right\|_{\beta \alpha_{s}^{*}} \\
& =C^{\frac{1}{\beta}+\frac{1}{\beta^{2}}}(\sqrt{1+\lambda})^{\frac{1}{\beta}+\frac{1}{\beta^{2}}} \beta^{\frac{1}{\beta}+\frac{2}{\beta^{2}}}\left\|\left|u_{\lambda}\right|\right\|_{\beta \alpha_{s}^{*}}
\end{aligned}
$$

Iterating this process and recalling that $\beta \alpha_{s}^{*}=2_{s}^{*}$, we conclude that for every $m \in \mathbb{N}$,

$$
\left\|\left|u_{\lambda}\right|\right\|_{\beta^{m} 2_{s}^{*}} \leq C^{\sum_{i=1}^{m} \frac{1}{\beta^{i}}}(\sqrt{1+\lambda})^{\sum_{i=1}^{m} \frac{1}{\beta^{i}}} \beta^{\sum_{i=1}^{m} \frac{i}{\beta^{i}}}\left\|\left|u_{\lambda}\right|\right\|_{2_{s}^{*}}
$$

Set $d_{m}=\sum_{i=1}^{m} \frac{1}{\beta^{i}}$ and $e_{m}=\sum_{i=1}^{m} \frac{i}{\beta^{i}}$. Then $d_{m} \rightarrow \sigma_{1}>0$ and $e_{m} \rightarrow \sigma_{2}>0$ as $m \rightarrow \infty$. Then, taking the limit in (2.8) as $m \rightarrow+\infty$, by Lemma 2.1 we have

$$
\left\|\left|u_{\lambda}\right|\right\|_{L^{\infty}} \leq C^{\sigma_{1}}(\sqrt{1+\lambda})^{\sigma_{1}} \beta^{\sigma_{2}} C:=B(1+\lambda)^{D}
$$

where $B:=C^{\sigma_{1}} \beta^{\sigma_{2}} C>0$ and $D:=\frac{\sigma_{1}}{2}$. This completes the proof.

Proof of Theorem 1.1 By Lemma 2.2, for large $M>0$, we can choose small $\lambda_{0}>0$ such that $\left\|\left|u_{\lambda}\right|\right\|_{L^{\infty}} \leq B(1+\lambda)^{D} \leq M$ for all $\lambda \in\left(0, \lambda_{0}\right]$. Consequently, $u_{\lambda}$ is a nontrivial solution of (1.1) with $\lambda \in\left(0, \lambda_{0}\right]$. This completes the proof.

\section{Acknowledgements}

We would like to thank the referees for their valuable comments and suggestions.

\section{Funding}

This work was supported in part by the National Natural Science Foundation of China $(11801153,11501403,11601145$, 11701322, 11901514), the Honghe University Doctoral Research Programs (XJ17B11, XJ17B12), the Yunnan Province Applied Basic Research for Youths (2018FD085), the Yunnan Province Local University (Part) Basic Research Joint Project (2017FH001-013), the Yunnan Province Applied Basic Research for General Project (2019FB001), Technology Innovation Team of University in Yunnan Province, and the Project funded by China Postdoctoral Science Foundation (2019M652790). 


\section{Competing interests}

The authors declare that they have no competing interests.

\section{Authors' contributions}

QL and JZ conceived of the idea of this manuscript and wrote the manuscript. KT and WW discussed about some estimation and checked the calculations. All authors read and approved the final manuscript.

\section{Author details}

'Department of Mathematics, Honghe University, Mengzi, P.R. China. ${ }^{2}$ Department of Mathematics, Taiyuan University of Technology, Taiyuan, P.R. China. ${ }^{3}$ Department of Mathematics and Statistics, Yunnan University, Kunming, P.R. China. ${ }^{4}$ School of Mathematics and Statistics, Hunan University of Technology and Business, Changsha, P.R. China. ${ }^{5}$ School of Mathematics and Statistics, Central South University, Changsha, P.R. China.

\section{Publisher's Note}

Springer Nature remains neutral with regard to jurisdictional claims in published maps and institutional affiliations.

Received: 4 March 2020 Accepted: 8 June 2020 Published online: 18 June 2020

\section{References}

1. Ambrosio, V., $d^{\prime}$ Avenia, P.: Nonlinear fractional magnetic Schrödinger equation: existence and multiplicity. J. Differ. Equ. 264, 3336-3368 (2018)

2. Antonelli, P., Athanassoulis, A., Hajaiej, H., Markowich, P.: On the XFEL Schrödinger equation: highly oscillatory magnetic potentials and time averaging. Arch. Ration. Mech. Anal. 211, 711-732 (2014)

3. Bonheure, D., Nys, M., Van Schaftingen, J.: Properties of ground states of nonlinear Schrödingder equations under a weak constant magnetic field. J. Math. Pures Appl. https://doi.org/10.1016/j.matpur.2018.05.007

4. d'Avenia, P., Squassina, M.: Ground states for fractional magnetic operators. ESAIM Control Optim. Calc. Var. 24, 1-24 (2018)

5. Di Cosmo, J., Van Schaftingen, J.: Semiclassical stationary states for nonlinear Schrödinger equations under a strong external magnetic field. J. Differ. Equ. 259, 596-627 (2015)

6. Di Nezza, E., Palatucci, G., Valdinoci, E.: Hitchhiker's guide to the fractional Sobolev spaces. Bull. Sci. Math. 136, 521-573 (2012)

7. Ding, Y., Wang, Z.: Bound states of nonlinear Schrödinger equations with magnetic fields. Ann. Mat. 190, 427-451 (2011)

8. Fournais, S., Treust, L.L., Raymond, N., Van Schaftingen, J.: Semiclassical Sobolev constants for the electro-magnetic Robin Laplacian. J. Math. Soc. Jpn. 69, 1667-1714 (2017)

9. Ji, C., Radulescu, V.D.: Multi-bump solutions for the nonlinear magnetic Schrödinger equation with exponentia critical growth in $\mathbb{R}^{2}$. Manuscr. Math. https://doi.org/10.1007/s00229-020-01195-1

10. Laskin, N.: Fractional quantum mechanics and Lévy path integrals. Phys. Lett. A 268, 298-305 (2000)

11. Laskin, N.: Fractional Schrödinger equation. Phys. Rev. E 66, 056108 (2002)

12. Li, Q., Teng, K., Wu, X.: Ground states for fractional Schrödinger equations with critical growth. J. Math. Phys. 59, $033504(2018)$

13. Li, Q., Wu, X.: Soliton solutions for fractional Schrödinger equations. Appl. Math. Lett. 53, 119-124 (2016)

14. Liang, S., Repovs, D., Zhang, B.: On the fractional Schrödinger-Kirchhoff equations with electromagnetic fields and critical nonlinearity. Comput. Math. Appl. 75, 1778-1794 (2018)

15. Reed, M., Simon, B.: Methods of Modern Mathematical Physics. IV. Analysis of Operators. Academic Press, London (1978)

16. Shang, X., Zhang, J.: Ground states for fractional Schrödinger equations with critical growth. Nonlinearity $27,187-207$ (2014)

17. Squasssina, M., Volzone, B.: Bourgain-Brézis-Mironescu formula for magnetic operators. C. R. Math. 354, 825-831 (2016)

18. Tang, X., Chen, S.: Ground state solutions of Nehari-Pohozaev type for Kirchhoff-type problems with general potentials. Calc. Var. Partial Differ. Equ. 56, 110 (2017)

19. Tang, X., Cheng, B.: Ground state sign-changing solutions for Kirchhoff type problems in bounded domains. J. Differ. Equ. 261, 2384-2402 (2016)

20. Xiang, M., Radulescu, V.D., Zhang, B.: A critical fractional Choquard-Kirchhoff problem with magnetic field. Commun. Contemp. Math. 21, 1850004 (2019)

21. Zhang, J., Zhang, W., Tang, X.: Ground state solutions for Hamiltonian elliptic system with inverse square potential. Discrete Contin. Dyn. Syst. 37, 4565-4583 (2017)

22. Zhang, J., Zhang, W., Xie, X.: Infinitely many solutions for a gauged nonlinear Schrödinger equation. Appl. Math. Lett. 88, 21-27 (2019)

23. Zhang, W., Zhang, J., Mi, H.: On fractional Schrödinger equation with periodic and asymptotically periodic conditions Comput. Math. Appl. 74, 1321-1332 (2017) 